\title{
Research on Simulation Maintenance Training of Control System
}

\author{
Qunshu Shen ${ }^{1, a}$, Zhao Yao ${ }^{2, b}$, Jiuchao Li3,c \\ 1,2,3 No.1,Huayuan Road,Changchun City,Jinlin Prov. China 130117 \\ a65934435@qq.com,b22162308@qq.com,c247658766@qq.com
}

\author{
Keywords: Training system; Equipment demand; Simulation
}

\begin{abstract}
Existing control system simulation maintenance training equipment aging, training methods, training content, equipment support can not keep up with the needs of training students in the new era, this paper plans to build a control system simulation maintenance training system, improve the simulation maintenance training equipment support needs and explore the simulation maintenance training law. In order to help students broaden the training channels in the daily learning process, simulated maintenance training has the advantages of openness, repeatability and low training cost.
\end{abstract}

\section{The Purpose and Meaning of the Study}

As a large-scale training institution in the field of mechanical equipment use and maintenance, there are more than 20 sets of maintenance training simulators for more than ten kinds of vehicles in our school, which basically cover all the common models in the field of mechanical equipment use and maintenance at this stage. Relying on these simulated training equipment, our laboratory has initiated a teaching and training mode featuring simulated teaching. At present, the training contents include the use and operation of control system, disassembly and assembly of components, maintenance and fault training. However, due to the lack of systematic research on equipment support construction, the number of supporting equipment is currently large. There are insufficient types, the construction of the training system is not perfect, and there is still a certain gap between the requirements of standardized training.

This paper mainly studies the methods and means of simulation maintenance training for control system, combines the existing maintenance training simulator, explores the construction of simulation maintenance training system under standardized training conditions, demonstrates the requirements of support equipment, studies the law of simulation maintenance training, and carries out the actual maintenance training. The whole process of training and teaching is transferred to simulated maintenance training and teaching, giving full play to the maximum benefit of simulated maintenance training.

\section{Main Contents of Research and Practice}

Build Simulation Maintenance Training System of Control System. With the development of the industrial revolution and the increasing demand of the market for skilled workers, a large number of new equipments have been put into the market. As a technical education college, the training task of the control system maintenance specialty is to train and meet the needs of the use and maintenance of the mechanical equipment control system under standardized conditions, and to use and maintain the equipment. Comprehensive development of maintenance and management, proficiency in the operation and use of control system support equipment and testing equipment, with strong troubleshooting ability and temporary repair ability, with the basic quality of control system maintenance technicians technical personnel. In order to acquire the above-mentioned ability, we must learn the theoretical maintenance knowledge of the corresponding models and possess the basic maintenance skills and the ability to analyze and remove the faults of the control system. The need for standardized training. Therefore, it is imperative to build a new simulation training system for control systems. 
Simulation maintenance training equipment upgrading. Under the new market demand, some models have been or will be withdrawn from the historical stage, so the new training system should be re-built according to heavy, medium-sized mechanical equipment maintenance training simulator. Some of the simulators need to be replaced by new maintenance training simulators which are closer to the market. Others need to be upgraded on the original basis to improve their functions and performance. The updated simulator model will cover the main equipment in the market at present and for a period of time to come. The simulated maintenance training in Colleges and universities will realize seamless connection with the actual work after graduation.

To develop training contents that meet the characteristics of control system simulation maintenance training. Compared with the actual training, the simulated maintenance training has its outstanding advantages and some irreplaceable shortcomings. Therefore, the training content must be formulated scientifically. The training contents are divided into pre-use inspection, first-level technical maintenance, second-level technical maintenance, third-level technical maintenance and so on. Pre-use inspection includes vehicle handover, vehicle condition registration and other items, the first-level technical maintenance corresponding to daily maintenance, the second-level technical maintenance corresponding to weekly maintenance, the third-level technical maintenance corresponding to seasonal maintenance, as well as the case-based maintenance, special maintenance and so on. Because the training simulator lacks the peripheral structure support compared with the actual equipment, part of the control system of the simulator belongs to the imitation knot. The performance indexes of the control system are different from the actual equipment. Therefore, the correctness of the function check and the method steps are emphasized in the maintenance contents at all levels, and the specific performance indexes are improved. The line is weakened.

Improve training mode. In the teaching of the original simulator control system, because the simulator itself has an open structure, it is more convenient to observe and learn, and because the number of related safeguard equipment is less, it can not be guaranteed on a large scale, so the teaching process organization often adopts the teacher-led way, the teacher operates and demonstrates, and the students observe and learn by the side. Practice. The trainees did not integrate into the training with their masters' attitude, and it was difficult to give full play to their subjective initiative. After the improvement, the trainees, as the organizers and subjects of the training, are responsible for the preparation of the training plan, the preparation of the training equipment, the storage of theoretical knowledge, the organization and implementation of the training process, the analysis and disposal of special circumstances, and the summary of the comments after the training. The teachers are there to guide and correct serious mistakes. By adopting this mode, on the one hand, the trainees'professional skills and the ability of combining theory with practice have been obviously improved, on the other hand, their organizational and training abilities as maintenance technicians have been improved, so that they can adapt to the job requirements as soon as possible.

System construction. In the original simulation maintenance training mode, due to lack of relevant system guarantee, the technical status of the simulator is difficult to be guaranteed reliably. Once the simulator fails, it is difficult to restore quickly and then affects the normal implementation of the training process. In the new control system simulation maintenance training system, vigorously strengthen the system construction, to provide a solid guarantee for the development of training. One is to establish a maintenance system; combine the maintenance system with the current college students'teaching classes according to the students' teaching characteristics; the teacher leads the professional students to take charge of the maintenance of all the simulators; actively apply for, purchase and develop various maintenance equipment through various channels; cancel the actual operation of the first-level maintenance to the second-level maintenance Maintenance and three-level maintenance are the main tasks. Regular checks are made on the control circuit of the simulator, lubrication and clearance adjustment are made on the mechanical part, quality tracking records of the simulator are established, the maintenance work is carried out to the people, and the maintenance system is carried out to the end. The second is to improve the use and management system; teaching and research room staff less, teaching more, it is difficult to do the same as the maintenance unit, the use of management mainly relies on teachers and classroom 
administrators to manage, each class before and after class teachers and classroom administrators to transfer registration, mainly relying on paper office model. The process of using, registering and repairing is registered through various forms and records. This management model has the following shortcomings: first, it wastes a lot of time and energy to fill in and manage these forms and records; second, it is difficult to preserve process data, easy to lose, confusion, unclear responsibility and other phenomena. Therefore, combined with the current intelligent campus construction work, the establishment of a new use and management system, through the construction of equipment and equipment information use management system, all equipment and equipment types, names, models, quantities, functions, teaching objects and other input systems to achieve dynamic management. Establish three different roles of the director of the teaching and research department, the teacher and the equipment manager, and endow them with corresponding functions. Any role can real-time view the relevant information and use of equipment and equipment, equipment application, approval, issuance, recycling process all through the network office, equipment and equipment to achieve full process life-cycle tracking, not only improve the efficiency of equipment and equipment, but also standardize the equipment management process.

Improve Control System Simulation Maintenance Training and Support Equipment Needs. A complete training system can not be separated from timely and adequate equipment support. With the proposed standardized training objectives, under the new control system simulation maintenance training mode, the existing support equipment has the following problems: First, lack of technical documentation support, there is no training program and practice guidance in line with the characteristics of simulator maintenance books; Second, the components of the teaching department have not been formed, so it is difficult to ensure the continuous training of the intensity training; Third, it is the poor function and versatility of in-situ testing equipment, small quantity, miscellaneous models, inconvenient maintenance of its own, and tedious operation steps.

Strengthen the construction of technical documentation system. According to the characteristics of simulated maintenance training, the teaching and research department organizes special forces to arrange the practice programs and instructions for all types of vehicles. The subjects, contents, methods and matters needing attention of simulated maintenance training for all types of vehicle control systems are specified in detail. The contents include operation of control systems, disassembly and assembly of components, performance testing, etc. During the whole process of troubleshooting, the performance difference between the simulator and the actual equipment is supplemented, and the simulator has been tried out in the college class and other training institutions, and has been trimmed three times according to the trial situation. Compared with the actual equipment, the simulator mainly simulates the main functions of the control system, and has some changes in the circuit design. By sorting out the technical documents of the simulator, combining the special circuit of the simulator with the circuit of the actual equipment, a fault circuit analysis atlas which accords with the actual situation of the simulator is made, which is convenient for the students to analyze and master the model. The characteristics and rules of the simulator fault.

Actively apply various channels to apply for teaching department components to form a large-scale support capability. It has been 14 years since the simulator of the teaching and research department was put into use. The components of the simulator may be out of place at any time. Some of the simulators do not have any components. Some of the simulators have a certain number of accessories, but they are analyzed according to the failure rules. The vulnerable parts which do not belong to this type of simulator are faced with the embarrassment that they can not be used in a short time if they are damaged. Combining with the research on the fault rules of the same period, the catalogue of vulnerable parts of different types of simulators is sorted out, and the relevant components are applied through different channels and means. At present, the two types of vehicle control systems have been equipped with three sets of teaching department components, and the other models have 1 to 2 sets of teaching department components respectively, which can basically meet the current several main types of vehicle simulation maintenance training.

Develop and purchase maintenance equipment to keep the simulator in good technical condition. Simulator maintenance equipment mainly includes special inspection tools and testing equipment. 
In addition to eliminating circuit faults, the normal operation of the simulator requires regular adjustment of the clearance between the mechanical components, and timely and accurate inspection of the simulator performance. This requires a special inspection tool matched with the simulator. Existing inspection tools are mainly used to service the simulator. The actual equipment, based on the performance inspection, is redesigned according to the actual structure of the simulator. At the same time, some mechanical devices are purchased from the factory to adjust the tooling, which can check and adjust part of the performance of the simulator. According to the characteristics of the control system, according to the classification of similar vehicles, a group of universal testing equipment can be purchased, which can be applied to many types of vehicles. At that time, a testing system covering all types of vehicle control system will be formed, providing a solid guarantee for quickly determining the fault point and shortening the time of removing obstacles.

Study the Simulation Training of Control System. With the adjustment of training content and training direction, the training direction of each specialty is focused on new equipment. The number of new equipment is difficult to guarantee the simultaneous teaching and training of each specialty, which seriously restricts the normal realization of teaching and training benefits. In order to achieve the past goal, only broaden the training channel is feasible. Simulated maintenance training as an important way to expand the channel, the study of its training law is conducive to better serve the actual teaching.

The performance is relatively stable, and the work is more reliable. All the simulators in the teaching and research room are stored in the professional classroom, mainly for the use of a single teaching and research room, management continuity is strong, its storage conditions are better than the actual installation, and is not affected by car bumps. The simulator adopts an open structure, on the one hand, it is convenient for students to observe, on the other hand, it overcomes the shortcomings of small space, poor lighting conditions, inconvenience in disassembly and inspection of components, time-consuming and labor-consuming maintenance. For example, the three-stage maintenance of the motor needs to be maintained, the need to remove the motor from the real car, because space constraints often take an hour or longer, and only 20 minutes on the simulator, the training efficiency has been greatly improved. In the actual training, the weather conditions have a greater impact on the need for special personnel to protect vehicles, training effective time is short, and the simulator is located indoors, regardless of day and night, rain and snow storms do not affect the normal operation. There are great hidden dangers in the real vehicle driving training of the control system. On the premise of reliable limit mechanism, the simulation of driving training scene can be achieved by using two-degree-of-freedom swing table, but the safety factor is greatly improved. Simulation maintenance training is easy to combine with virtual reality technology. Virtual reality technology integrates the latest development of computer graphics technology, computer simulation technology, artificial intelligence, sensor technology, display technology, network parallel processing technology, etc. It can be widely used in principle teaching, use and maintenance, component repair, troubleshooting stages. Once the two are combined, the teaching methods will be greatly enriched. Its multi-perception, interaction, immersion, strong imagination and even the use of practical equipment training can not be achieved.

\section{Research Process and Method}

Collect training methods, training methods and common problems of equipment simulation maintenance training in factories and training institutions through the investigation of factories and training institutions and the feedback of trainees.

Collect the results of the investigation and feedback from the students, scientifically formulate the teaching content, compile the practice plan and practice instruction, try out the equipment support standard, and carry out the simulation maintenance training teaching of the control system.

According to the teaching situation, the internal discussion of the research group, the development of internship program, practice guidance, equipment support standards.

Verify the effect of teaching reform according to the assessment and questionnaire. According to 
the actual vehicle assessment model and the characteristics of simulation training, the assessment is organized to highlight the technical performance evaluation of equipment after repair, and the students'knowledge, ability and skill level are comprehensively inspected.

\section{Innovation Point}

Control system, real vehicle maintenance training, the whole process of simulation teaching. Control system simulation maintenance training subjects set close to the job needs of students, covering the whole process of control system vehicle maintenance training, the establishment of a unified and perfect teaching norms, improve the efficiency of simulation training equipment, teaching role is fully reflected.

Construct the simulation maintenance training system of control system, set up the matching standard of simulation maintenance training support equipment, and provide reference for the simulation training construction of related training institutions in factories.

The simulation maintenance training law of control system is explored, and the simulation teaching and training of control system is guided, and the training benefit is improved.

\section{Conclusion}

At present, the teaching classes for junior college students in $2016 \sim 2018$ have been applied. In the course of control system maintenance, the effect of simulated maintenance training is verified. The application shows that the simulated maintenance training can help the students master the whole process of comprehensive repair as well as the actual equipment training. The teaching content is more vivid and intuitive, the operation process is simple, the measurement results are accurate, and the teaching effect is improved.

\section{References}

[1] D.L. Zuo, X.M. Li: Experimental Technology and Management, ( 2017) No.10,P146(In Chinese)

[2] Z. Zhao: Time Report, (2018) No.4,P193(In Chinese)

[3] Y. Chen, F.Z. Fu: Higher Engineering Education Research,（2008） No.1,P47(In Chinese)

[4] J.N. Huang. Off Campus Education in China, (2008) No.S1,P1304(In Chinese)

[5] C.Y. Wu: Higher Vocational Education, (2016) (In Chinese)

[6] S.F. Shan: Guide for Education, (2014) No.19,P184(In Chinese) 